\title{
Coordination Structures in a Typed Feature Structure Grammar: Formalization and Implementation
}

\author{
Jong-Bok Kim ${ }^{1}$ and Jaehyung Yang ${ }^{2}$ \\ ${ }^{1}$ School of English, Kyung Hee University, Seoul, 130-701, Korea \\ jongbok@khu.ac.kr \\ ${ }^{2}$ School of Computer Engineering, Kangnam University, Kyunggi, 446-702, Korea \\ jhyang@kangnam.ac.kr
}

\begin{abstract}
Every language employs its own coordination strategies, according to the type of coordinating marking, the pattern of marking, the position of the marker, and the phrase types coordinated. The SOV language Korean is intriguing in the sense that it displays almost all the possibilities of these dimensions. This paper shows how a typed feature structure grammar, HPSG, together with the notions of 'type hierarchy' and 'constructions', can provide a robust basis for parsing the coordination constructions found in the language. We show that this system induces robust syntactic structures as well as enriched semantic representations for real-time applications such as machine translation, which require deep processing of the phenomena concerned.
\end{abstract}

\section{Basic Data: Two Main Types of Coordination}

Korean employs two kinds of coordination marking: morphological and lexical marking 1 In the morphological marking system, the language distinguishes nominal and verbal coordination. As seen in the corpus example (1a), nominal coordination uses suffixal markers (usually called particles in the traditional literature) like -(k)wa, -hako, -(i)lang 'and' for conjunctive and -(i)na 'or' for disjunctive coordination. Meanwhile, as in (1b), verbal coordination uses the suffixal marker - $k o$ 'and' for conjunctive and -kena 'or' for disjunctive coordination 2

\footnotetext{
${ }^{1}$ Our thanks go to three anonymous reviewers for the helpful comments and suggestions. This work was supported by the Korea Research Foundation Grant funded by the Korean Government (KRF-2005-042-A00056).

${ }^{2}$ The abbreviations for the glosses and attributes used in this paper are ACC (ACCusative), ARg (ARGUMENT), CARG (CONStant ARGUMENT), C-ARG (CONJunCt ARGUMENT), C-CONT (CONSTRUCTIONAL CONTENT), DAT (DATIVE), DECL (DEClARATIVE), HON (HONORIFIC), LBL (LABEL), L-INDEX (LEFT INDEX), LTOP (LOCAL TOP), NOM (NOMinATIVE), PNE (PRENOMINAL ENDing), PL (PLURAL), PST (PAST), R-INDEX (RIGHT INDEX), RELS (RELATIONS), TOP (TOPIC), etc.
} 
(1) a. [khempwuthe-wa/hako/ilang intheneys-ul] paywu-ess-ta computer-and internet learn-PST-DECL '(He) learned computer and internet.'

b. pelley-ey [mwulli-ko/kena sso-yess-ta] insect-DAT bite-and/or stung '(He) was bitten and/or stung by an insect.'

In addition to these morphological markers, the language has words like kuliko 'and', ttonun 'or' as lexical coordinators. Unlike the morphological coordinators, these coordinators can be used for both nominal and verbal coordination:

(2) a. hay-wa tal kuliko sem-i hamkkey ha-nun kos sun-and moon and island-NOM together do-PNE place 'the place where sun, moon, and island exist together'

b. mak-kena ttonun phihal swuissta

block-or or avoid can

'(You) can block or avoid it.'

In terms of the patterns of coordination marking, natural languages employ four main types of coordination constructions from asyndeton (with no marking in each conjunct) to omnisyndeton (with one marking for each conjunct) (cf. [1]):

(3) a. Asyndeton: A B C

b. Monosyndeton: A B conj C

c. Polysyndeton: A conj B conj C

d. Omnisyndeton: A conj B conj C conj

Our corpus search reveals that Korean displays all these types in spoken and written texts. We inspected the Sejong Treebank Corpus to check the possible patterns of Korean coordination. The corpus consists of 378,689 words $(33,953$ sentences). We identified total 6,345 instances of nominal coordination within which we identified all these four types. In particular, the following present the 5,378 instances of top 8 frequent patterns with maximum three conjunts we found in the corpus 3

(4)

\begin{tabular}{||l|l||l|l||}
\hline Patterns & Frequency & Patterns & Frequency \\
\hline \hline A(-)and B (mono) & 3,201 & A B(-)and, C (mono) & 167 \\
A(-)or B (mono) & 860 & A(-)and B(-)and C (poly) & 70 \\
A, B (asyndeton) & 508 & A-and B, C (mono) & 27 \\
A, B, C (asyndeton) & 534 & A-and B-and (omni) & 11 \\
\hline
\end{tabular}

As shown here, the language uses monosyndeton strategies most often. The corpus also reveals more asyndeton instances than polysyndeton or omnisyndeton.

There has been much debate regarding the syntactic structures of coordination. Among the central questions are whether it allows $n$-ary structures; and

\footnotetext{
3 The coordinators with a hyphen are the morphological ones whereas those with no
} hyphen are the lexical ones. 
which of the conjuncts serves as the head of the coordination phrase (cf. [2, 1]). Engineering considerations in our project have indicated that Korean requires both binary and ternary structures (cf. 2]). The descriptive facts also indicate that the final conjunct functions at least as the syntactic head of the coordination phrase. This paper provides an account of how these two basic assumptions, together with appropriate constructional constraints, can bring us an efficient and robust grammar for parsing the intriguing syntactic as well as semantic aspects of Korean coordination.

\section{Implementing an Analysis}

Needless to say, theoretical and engineering considerations lead us to prefer fewer rules in dealing with all these different types. Empirical data and our implementation results indicate that the most economic way of implementing the analysis in a typed feature structure grammar is to introduce the notion of constructional constraints within a multiple inheritance type hierarchy system.

\subsection{Lexical Information}

In monosyndeton strategies, as noted, the language can use either morphological marking or a lexical coordinator:

(5) a. A-and/or B salam-kwa/-ina cimsung ('human and/or animal')

b. A and/or B yokmang kuliko/ttonun pwulan ('desire and/or anxiety')

The attachment of a morphological marker like -kwa or -ina onto a nominal differentiates it from a canonical nominal and introduces the head feature COORD. The need to treat this as a head feature comes from complex examples in which the marking information needs to pass up to the mother NP:

(6) $\left[\left[N_{N}[\right.\right.$ nelp-un cip-kwa $] \quad\left[N_{N}[\right.$ alumtaw-un cengwon-ul $\left.]\right]$ calanghayssta wide-PNE house-and pretty-PNE garden-ACC boasted 'It boasted a wide house and beautiful garden.'

The lexicon thus adds the head feature COORD to a nominal or verbal expression when it hosts a morphological coordination marker:
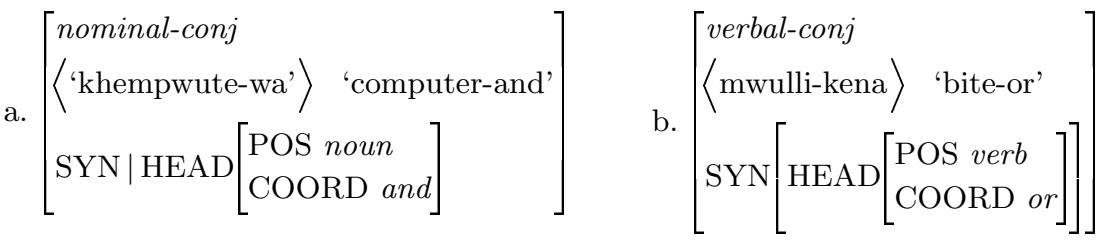

The lexical coordinators are no different. Just like the morphological markers, our grammar takes these words to provide the COORD value, as exemplified in (8): 
(8)
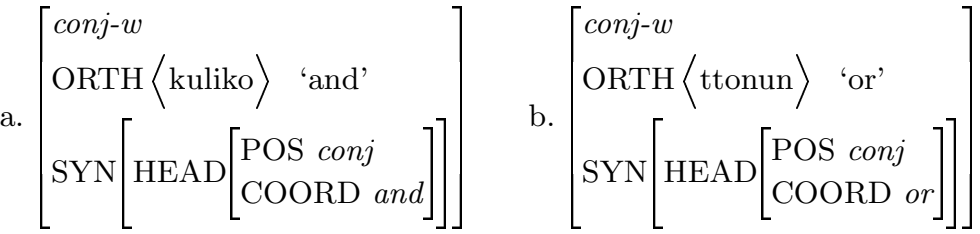

\subsection{Syntactic Aspects}

Different from other languages, Korean coordination appears to assign the syntactic headedness to the last conjunct. For example, the (nominative) CASE or HON value of a nominal coordination and the MOOD value of verbal coordination are projected from the final conjunct.

(9) a. [[haksayng-kwa] [sensayng-nim-i]] o-si-ess-ta

student-and teacher-HON-NOM come-HON-PST-DECL

'Students and teachers came.'

b. [[namca-nun o-ass-ko] [yeca-un ttena-ss-ta]]

men-TOP come-PST-and women leave-PST-DECL

'Men came, and women left.'

However, the coordinated phrases need to be like categories. In particular, the conjuncts need to have the same POS (part of speech) and VAL (valence) values:

(10) a. ${ }_{N P}\left[\right.$ haksayng-kwa] ${ }_{A d v P}[$ ppalli] o-ass-ta
student-and
fast come-PST-DECL
b. ${ }^{*}{ }_{S / N P}\left[\right.$ haksayng-un _ ilk-ess-ko] $\quad{ }_{S}[$ sensayng-nim-un
student-TOP read-PST-and teacher-HON-HON
hayngpokha-n] chayk]
happy book
'*the book that students read and teachers were happy'

As shown in (10a), we cannot coordinate an NP with an AdvP. And as shown in (10b), an $\mathrm{S}$ with a gap cannot be coordinated with a fully saturated $\mathrm{S}$ since they have different VAL values 4

Our grammar contributes the following general constraints to the coordination construction defined as coord-ph, in terms of a grammar rule:

Coordination Rule:

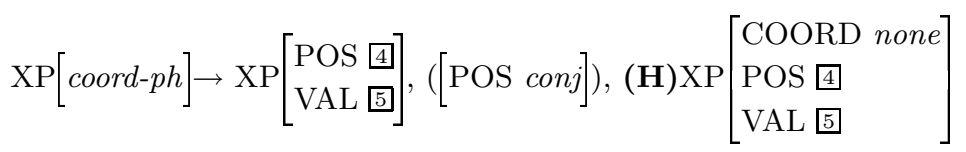

The rule in (11), which all instances of coordination need to observe, basically says that two identical XPs can be conjoined when they share POS and VAL values, while the last conjunct serves as the syntactic head. The first conjunct

\footnotetext{
${ }^{4}$ Valence values here include subject, complements, and slashed elements.
} 
has no constraint on the value of COORD5 5 , yet the last conjunct bears the head feature [COORD none] value. This will block a coordination-marked phrase from appearing in a final conjunct 6

Depending on the appearance of the second conjunction word, the phrase will be realized either as a binary structure bin-coord-ph or as a ternary structure tern-coord-ph. When the middle element (lexical coordinator) of the phrase is absent, we license a bin-coord-ph with patterns like (12). Meanwhile, when the conjunction word occurs, we will have a tern-coord-ph with patterns like (13):

(12) a. A, B

b. A-and B

c. A-or B
(13) a. A and/or B

b. A-and and B

c. A-or or B

When bin-coord-ph and tern-coord-ph are combined, we will have various patterns of coordination, some of which are as follows:
(14) a. A, [B and $\mathrm{C}]$
b. A, [B-and and $\mathrm{C}]$
d. A, $[\mathrm{B}, \mathrm{C}]$
c. $[\mathrm{A}$-and $[\mathrm{B}$ and $\mathrm{C}]]$
e. [A-and [B-and and $\mathrm{C}]]$
f. $[\mathrm{A}$-and $\mathrm{B}]$ and $[\mathrm{C}$-and $\mathrm{D}]$

Notice that the language also uses omnisyndeton strategies in which all conjuncts are marked with a coordination marker. Omnisyndeton is possible only with the morphological coordinators -hako and -(i)lang:

(15) a. kongchayk-hako/ilang yenphil-hako/ilang ciwukay-hako/ilang sassta.

$$
\text { notebook-and pencil-and eraser-and bought }
$$

'(I) bought notebooks, pencils, and erasers.'

b. *kongchayk-kwa yenphil-kwa ciwukay-wa sassta.

notebook-and pencil-and eraser-and bought

The final conjunct with marking hako or ilang thus functions just like a canonical NP with no coordination marking. To deal with this, we assume that the nominals with such a marking have an underspecified COORD value:

(16)

$$
\left[\begin{array}{l}
\text { nominal-ilang-hako } \\
\text { SYN | HEAD }\left[\begin{array}{l}
\text { POS noun } \\
\text { COORD and-none }
\end{array}\right]
\end{array}\right]
$$

This lexical information means that words like ciwukay-hako 'eraser-and' or ciwukay-lang can be either [COORD none] or [COORD and].

\footnotetext{
${ }^{5}$ The subtypes of coord-ph can place constraints on the COORD value.

${ }^{6}$ The value of [COORD coord] is defined as follows:
}

(i) a. coord: and-none, or-none

b. and-none: and, none

c. or-none: or, none 


\subsection{Semantic Aspects and Constructional Constraints}

In deep-processing the coordination structures, complications arise in how to get the appropriate semantics ([3]). We can simply assume that the morphological or lexical coordinator (marked with COORD feature) determines the conjunctive or disjunctive meaning of a coordination phrase. One issue arises from doubly-marked phrases. As noted before, in coordinating two NPs, we can have both the morphological marking - wa as well as the lexical conjunction word kuliko:

(17) hyencay-(wa) kuliko/ttonun milay-lul sayngkakhay poca. present-and and future-ACC think let 'Let's think about the present and future!'

If each of these morphological and lexical markers induce its own independent semantic relation, we would have too many coordination relations: one at least is redundant. Another issue lies in asyndeton strategies in which no marking appears:

(18) haksayng, hakpwumo, kyosa-tul-i chamsekhayessta

student parent teacher-PL-NOM attended

'Students, parents, and teachers attended.'

In such an example, even though we have only a conjunctive reading, the question arises as to what triggers this meaning.

These observations, together with our trial and error progress from implementations, led us to make the supposition that the coordination relation is invoked as a constructional meaning, represented in (19):

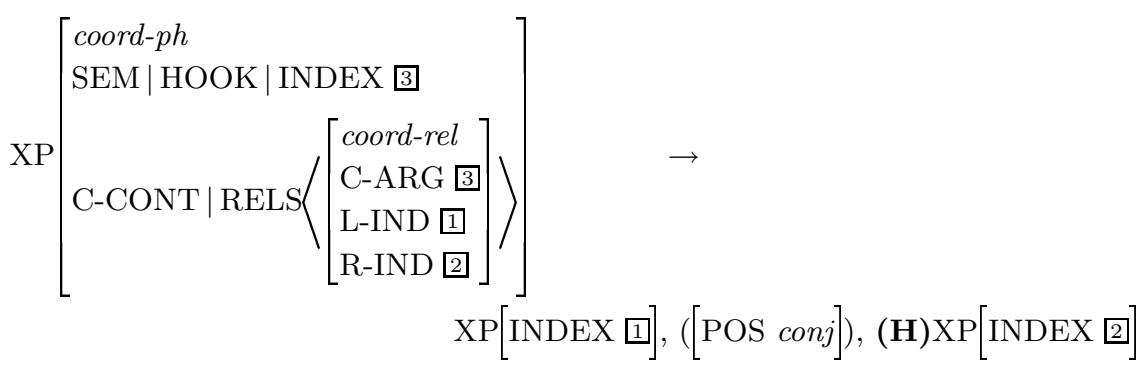

The semantic (SEM) information of the phrase, represented in the format of MRS (Minimal Recursion Semantics), includes an INDEX value. In addition, we can see here that the coord-ph introduces a constructional relation coord-rel in the C-CONT (constructional content). This relation has three arguments: C-ARG (conjunct argument), L-INDEX (left conjunct's index) and R-INDEX (right 
conjunct's index value). The value of C-ARG is the conjoined index conj-index which serves as a pointer to the separate conjoined entity and thus is identified with the INDEX value of the whole phrase 7

The question that follows is then how we can distinguish conjunctive from disjunctive coordination. In answering this, the grammar classifies coord-ph into two dimensions as represented in the following multiple inheritance hierarchy:

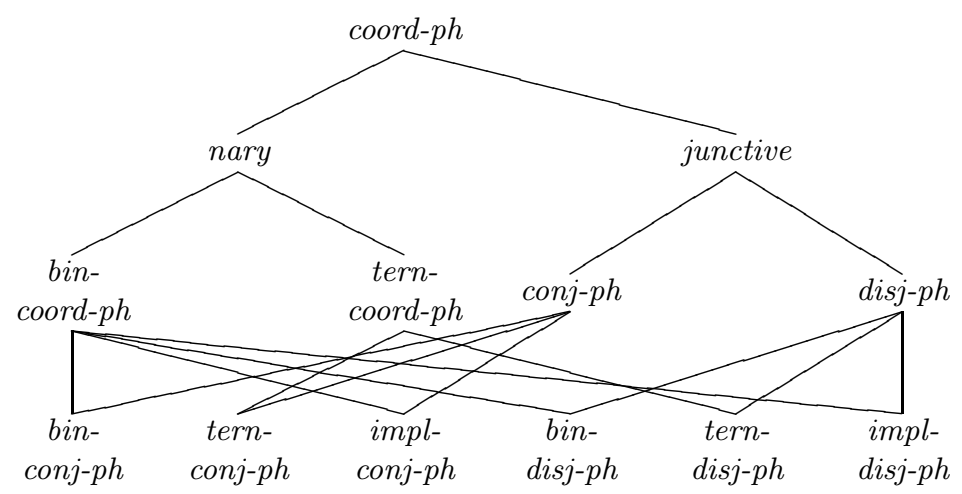

Each type has its own syntactic as well as semantic constraints, capturing the generalizations among types. Any constraints on a supertype will be inherited to its subtypes. The types bin-coord-ph and tern-coord-ph will determine the syntactic structure of the conjunct daughters as we have seen before. Meanwhile, the phrases conj-ph and disj-ph introduce a conjunctive or disjunctive relation in the C-CONT:

$$
\begin{aligned}
& \text { a. } \mathrm{XP}\left[\begin{array}{l}
\text { conj-ph } \\
\mathrm{C}-\mathrm{CONT} \mid \mathrm{RELS}\langle[\text { and_coord_re }]]\rangle
\end{array}\right] \rightarrow \mathrm{XP}, \ldots \\
& \text { b. } \mathrm{XP}\left[\begin{array}{l}
\text { disj-ph } \\
\left.\mathrm{C}-\mathrm{CONT} \mid \mathrm{RELS}\langle[\text { or_coord_rel } l]\rangle_{]}\right] \rightarrow \mathrm{XP}, \ldots
\end{array}\right.
\end{aligned}
$$

Meanwhile, their subtypes specify which conjunct daughter contributes this coordination meaning together with the constraints on the COORD value:

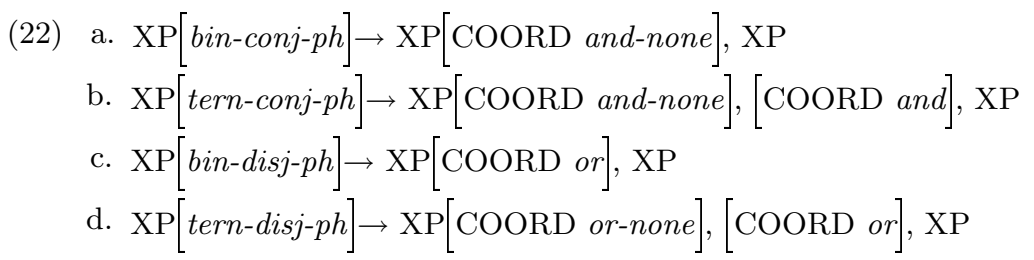

\footnotetext{
${ }^{7}$ Minimal Recursion Semantics, developed by 4, is a framework of computational semantics designed to enable semantic composition using only the unification of type feature structures. See 4] and [5] The value of the attribute SEM(ANTICS) in our system represents a simplified MRS.
} 
In bin-conj-ph and bin-disj-ph, the first conjunct determines the coordination meaning whereas in tern-conj-ph and tern-disj-ph, the second element (conjunction word) regulates the meaning.

There are two additional things to be noted here. First, note that the value of COORD in the first conjunct of tern-conj-ph and tern-disj-ph is and-none and or-none, implying its value can be either and/or or none. This constraint on the COORD value allows the grammar to license the symmetric patterns in (23) but not the asymmetric patterns in (24), when we have both the morphological and lexical coordinators 8

(23) a. A-(and) and B b. A-(or) or B
(24) a.*A-(or) and B b. ${ }^{*} \mathrm{~A}-($ and $)$ or $\mathrm{B}$

Second, notice the COORD value of the first conjunct in bin-conj-ph and bindisj-ph. It is and-none in the former whereas it is or in the latter. This ensures that the asyndeton strategies will induce only a conjunctive reading:

(25) [kunsim, kekceng-i] epsi cal cinaywassta

concern anxiety without well spent

'(I) have been well without worry and anxiety.'

In bin-conj-ph, the first conjunct's COORD value is and-none. When its value is none, the grammar can license examples like (25). Even though the first conjunct has no marking, the combination of kunsim, kekceng here will form a bin-conj-ph with a and_coord_rel. There is no rule that induces a disjunctive reading for such a case, as proved from the parsing results too. Our grammar is thus restrictive in the sense that when there is no coordination marking at all as in (25), we have a conjunctive reading only.

However, a complication arises here from examples in which the interpretation of the top coordination phrase with no coordinator depends on the type of the lower coordination phrase. Consider the following:

(26)
a. [si, [kulim-(kwa) (kuliko) iyaki-ka]] iss-nun kos poem picture and story exist-PNE place 'the place where poems, pictures, and stories exist.'
b. [si, [kulim-(ina) (ttonun) iyaki-ka]] iss-nun kos poem picture or story exist-PNE place 'the place where poems, pictures, or stories exist.'

The example (26a) induces only a conjunctive reading, whereas (26b) only a disjunctive reading 9 In order to capture these constraints, we have two additional types of coordination as the subtypes as noted in the hierarchy (20):

\footnotetext{
${ }^{8}$ Our Google web search reveals less than 100 instances of such asymmetric coordination patterns. If such examples are really acceptable, we simply need to remove the constraints on the value of the attribute COORD in the first conjunct.

${ }^{9}$ A flat structure analysis may solve such an issue, but as noted by [1, it will require a great deal of grammar rules in the implementation.
} 
(27)

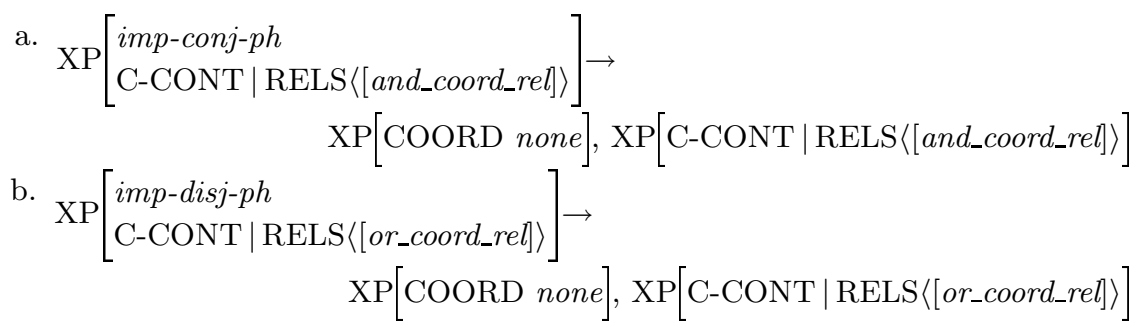

These rules will allow us to induce appropriate semantics for the different asyndenton strategies such as "A, [B and C]" (only conjunctive reading) and "A, [B or C]" (only disjunctive reading).

\section{Results of the Implementation}

The analysis we have presented so far has been incorporated in the typed-feature structure grammar HPSG for Korean (Korean Resource Grammar) aiming at working with real-world data (cf. 6] and [7]). To test its performance and feasibility, it has been implemented into the LKB (Linguistic Knowledge Building) 10 The test results give the proper syntactic as well as semantic structures for all the coordination patterns from simple binary or ternary to complex patterns we find in the language.

For example, (28) is the syntactic and MRS structure for the example si-wa kulim-kwa kuliko iyaki 'poem-and, picture-and and story' where the morphological marker - wa and the lexical coordinator kuliko occur together. In terms of the syntactic structures, the grammar generates only one structure for the NP as given in the output here: kulim-kwa kuliko iyaki forms a tern-conj-ph and then this resulting phrase will form a bin-conj-ph with si-wa 11 We can notice here that the MRS the grammar generates provides enriched information of the phrase. The value of LTOP is the local top handle, the handle of the relation with the widest scope within the constituent. The attribute RELS is basically a bag of elementary predications (EP) each of whose value is a relation 12 Each of the types relation has at least three features LBL, PRED (represented here as a type), and ARG0. The INDEX value here is identified with the ARG0 (C-ARG) value of the first and_rel within the RELS list here. The L-INDEX value of this relation is identified with the udef_q_rel for the noun poem that serves as the first conjunct 13 The R-INDEX value is iden-

10 The current Korean Resource Grammar has 394 type definitions, 36 grammar rules, 77 inflectional rules, 1100 lexical entries, and 2100 test-suite sentences, and aims to expand its coverage on real-life data.

11 The system does not combine si-wa with kulim-kwa first since the latter is marked with [COORD and], which would violate the constraint on coord-ph.

12 The attribute HCONS is to represent quantificational information. See [5].

${ }^{13}$ Korean common nouns do not require a determiner to project an NP. Even though a determiner is not available, we need to express an underspecified quantification on the noun in order to make the semantics compatible with the semantic output of other languages, and to make scope restrictions work. Such a move is essential in deep processing aimed at multilingual applications. 
tified not with any conjunct but with the ARG0 of the other and_rel representing the semantics of kulim-kwa kuliko iyaki 'picture and story'.

(28)

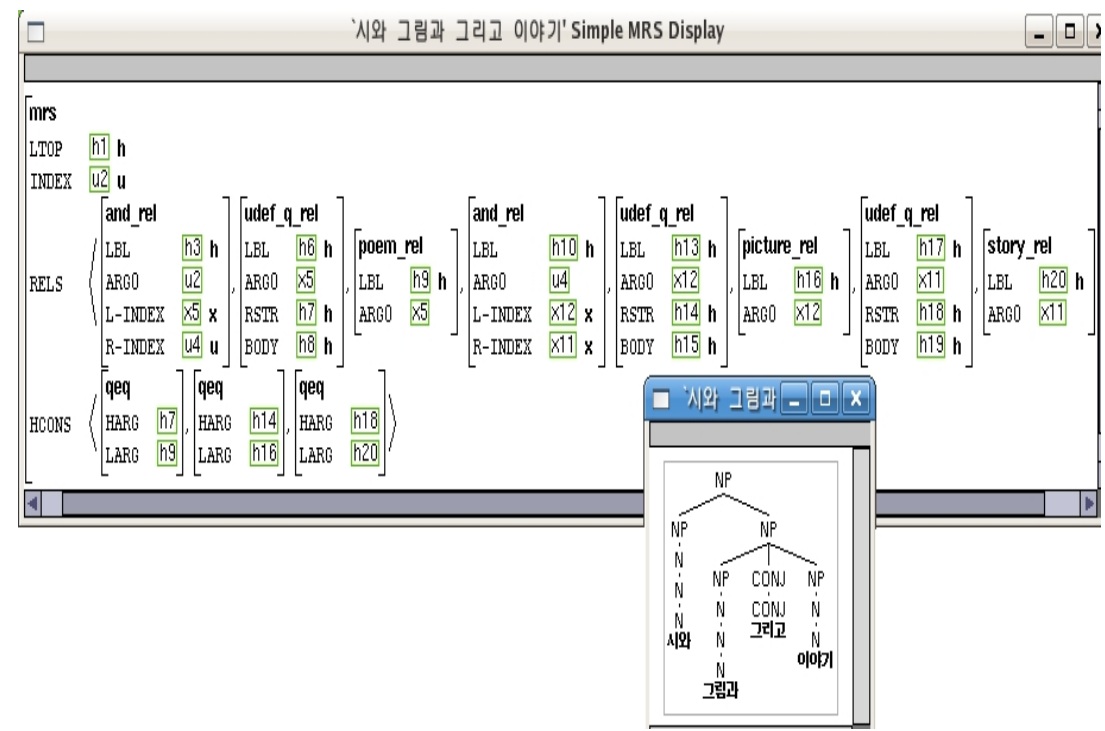

Now let's look at a combination of asyndeton and monosyndeton, si, kulim kuliko iyaki 'poem, picture and story':

(29)

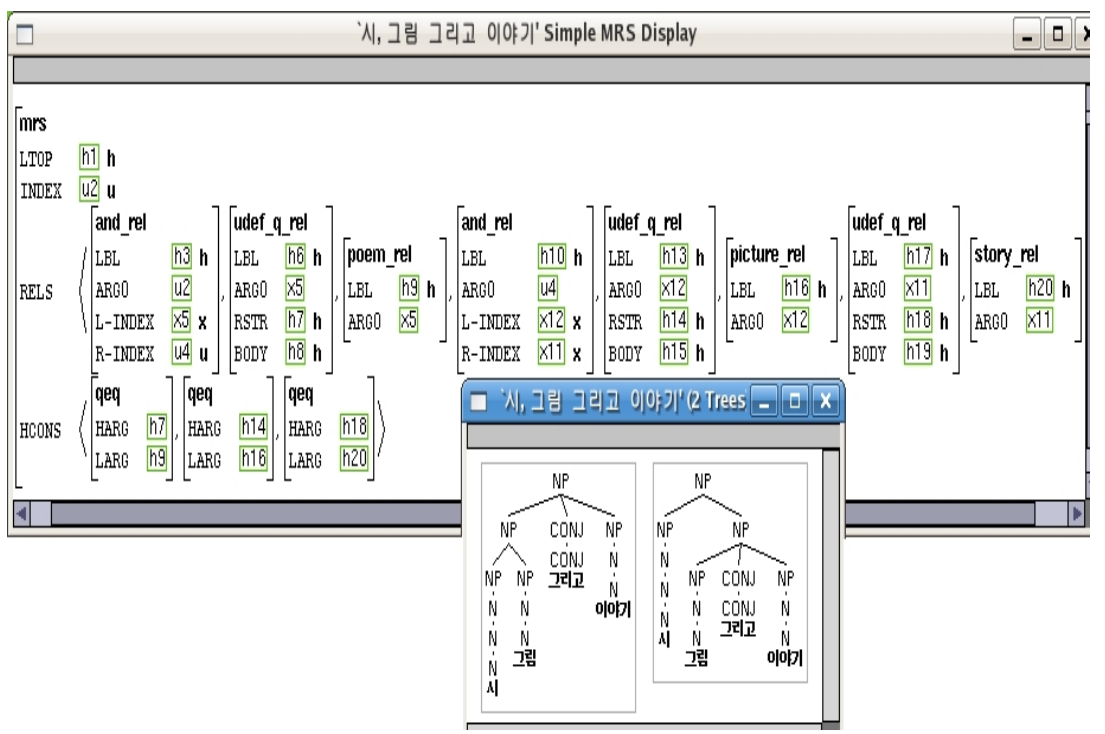

As noted in the parse trees, we have two syntactic structures. In the first tree $s i$ 'poem' combines with kulim 'picture' as an impl-conj-ph and the result forms 
a tern-conj-ph together with the conjunction word kuliko and iyaki 'story'. In the second tree kulim, kuliko and iyagi forms a tern-conj-ph first and then forms an impl-conj-ph with si. The MRS here represents the meaning of the second tree. One thing to note here is that even though there is neither morphological marking nor lexical marking, the constraint on impl-conj-ph induces only a conjunctive reading (adding a and_rel).

Our system also allows the appropriate syntactic as well as semantic representations for the omnisyndeton coordination. Consider the parsing results of the example kongchayk-ilang, yenphil-ilang, ciwukay-lang 'notebook-and, pencil-and, eraser-and':

(30)

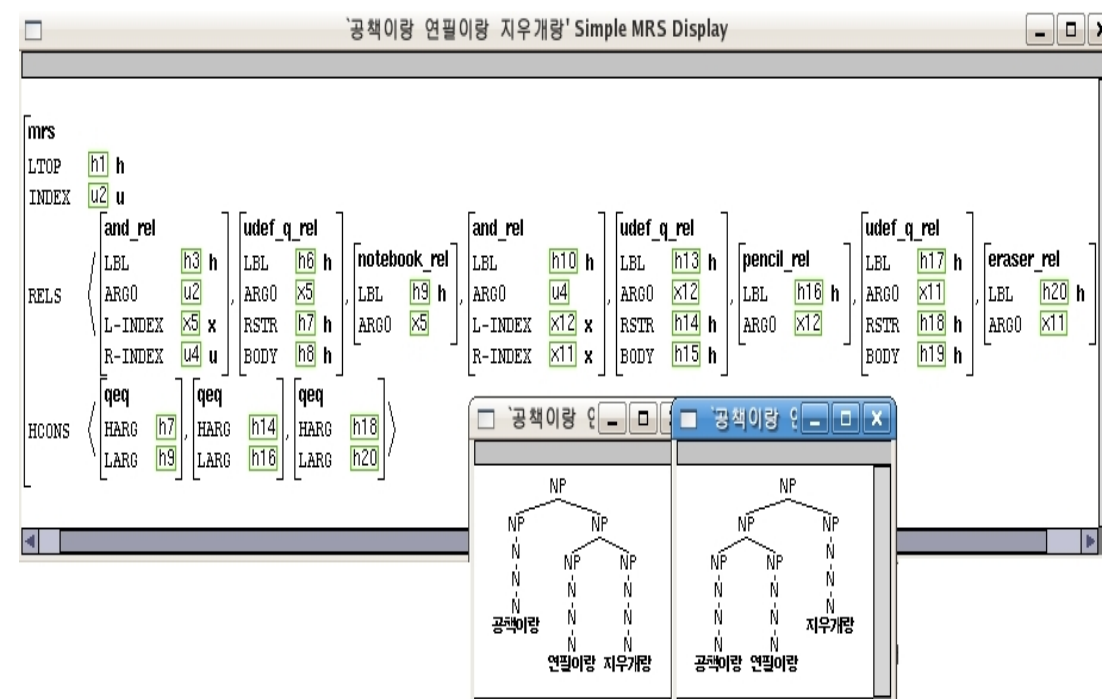

Once again, we have two possible structures depending on the order of combining the conjuncts (each combination forms a bin-conj-ph here). Even though the final conjunct is marked with -ilang, our lexical specification in (16) allows it to have [COORD none]. Also note that our constructional approach invokes no redundant coord_rel, proving the efficiency of the grammar.

\section{Conclusion}

As noted earlier, coordination phrases have a high frequency in real-life texts. In the present analysis, the grammatical constraints are encoded in the multiple inheritance hierarchy where the subtypes of coordination phrases are arranged. This allows us to capture both syntactic and semantic generalizations across all of the different coordination constructions in a systematic way.

Any grammar, aiming for real-world applications, needs to provide a correct syntax from which we can build semantic representations in compositional 
ways. In addition, these semantic representations must be rich enough to capture compositional as well as constructional meanings. In this respect, the analysis we have sketched here seems to be promising as it provides enriched semantic representations for various types of coordination that should be suitable for applications requiring deep natural language processing.

\section{References}

1. Drellishak, S., Bender, E.M.: A coordination module for a crosslinguistic grammar resource. In Müller, S., ed.: The Proceedings of the 12th International Conference on Head-Driven Phrase Structure Grammar, Department of Informatics, University of Lisbon, Stanford, CSLI Publications (2005) 108-128

2. Abeillé, A.: A lexicon- and construction-based approach to coordinations. In Müller, S., ed.: Proceedings of the HPSG-2003 Conference, Michigan State University, East Lansing, Stanford, CSLI Publications (2003) 5-25

3. Bender, E.M., Siegel, M.: Implementing the syntax of Japanese numeral classifiers. In: Proceedings of IJCNLP-04. (2004)

4. Copestake, A., Flickenger, D., Sag, I., Pollard, C.: Minimal recursion semantics: An introduction. Manuscript (2003)

5. Bender, E.M., Flickinger, D.P., Oepen, S.: The grammar matrix: An open-source starter-kit for the rapid development of cross-linguistically consistent broad-coverage precision grammars. In Carroll, J., Oostdijk, N., Sutcliffe, R., eds.: Proceedings of the Workshop on Grammar Engineering and Evaluation at the 19th International Conference on Computational Linguistics, Taipei, Taiwan (2002) 8-14

6. Kim, J.B., Yang, J.: Projections from morphology to syntax in the korean resource grammar: implementing typed feature structures. In: Lecture Notes in Computer Science. Volume 2945. Springer-Verlag (2004) 13-24

7. Kim, J.B.: Korean Phrase Structure Grammar. Hankwuk Publishing, Seoul (2004) In Korean. 